\title{
Physics potential of a muon-proton collider
}

\author{
Kingman Cheung $\odot^{1,2, *}$ and Zeren Simon Wang $\oplus^{2, \dagger}$ \\ ${ }^{1}$ Department of Physics, Konkuk University, Seoul 05029, Republic of Korea \\ ${ }^{2}$ Department of Physics, National Tsing Hua University, Hsinchu 300, Taiwan
}

(Received 1 February 2021; accepted 9 May 2021; published 8 June 2021)

\begin{abstract}
We propose a muon-proton collider with asymmetrical multi-TeV beam energies and integrated luminosities of $0.1-1 \mathrm{ab}^{-1}$. With its large center-of-mass energies and yet small Standard Model background, such a machine can not only improve electroweak precision measurements but also probe new physics beyond the Standard Model to an unprecedented level. We study its potential in measuring the Higgs properties, probing the R-parity-violating supersymmetry, as well as testing heavy new physics in the muon $g-2$ anomaly. We find that for these physics cases the muon-proton collider can perform better than both the ongoing and future high-energy collider experiments.
\end{abstract}

DOI: 10.1103/PhysRevD.103.116009

\section{INTRODUCTION}

The Large Hadron Collider (LHC) in Geneva, Switzerland, has culminated with the discovery of a Standard Model (SM)-like Higgs boson in 2012 [1,2]. However, the world's largest machine has so far failed to find any new fundamental particles predicted by models beyond the Standard Model (BSM), e.g., the sleptons and squarks proposed by supersymmetry (SUSY) models $[3,4]$. While the LHC program will be finished only in mid 2030's, a wide range of next-generation colliders have been proposed and intensively discussed. For example, various $e^{+} e^{-}$ colliders to run as a Higgs or Z-boson factory, have been suggested, including the International Linear Collider (ILC) [5,6], Compact Linear Collider (CLIC) [7], the Future Circular Collider in the ee mode (FCC-ee) [8], and the Circular Electron Positron Collider (CEPC) [9]. Then, there are two main electron-proton collider proposals, i.e., the Large Hadron-electron Collider ( $\mathrm{LHeC}$ ) [10-13] and FCC in the hadron-electron mode (FCC-he) [14,15], to be running concurrently with and after the high-luminosity LHC (HL-LHC), respectively. In addition to these proposals, there are also higher-energy proton-proton colliders: High Energy LHC (HE-LHC) [16-18], FCC in the hadron-hadron mode (FCC-hh) [14,15], and Super Proton Proton Collider (SPPC) [19], as well as renewed interests in muon colliders [20-32]. ${ }^{1}$ The experiments in these colliders would, for

\footnotetext{
*cheung@phys.nthu.edu.tw

†wzs@mx.nthu.edu.tw

${ }^{1}$ More recently electron-muon head-on collisions have also been studied for the first time in Refs. [33,34].

Published by the American Physical Society under the terms of the Creative Commons Attribution 4.0 International license. Further distribution of this work must maintain attribution to the author(s) and the published article's title, journal citation, and DOI. Funded by SCOAP ${ }^{3}$.
}

instance, excel in electroweak (EW) precision measurements, quantum chromodynamics (QCD) tests, or BSM physics searches.

In this work, we consider a muon-proton collider with multi-TeV beam energies. Historically this type of machine was first investigated in late 1990's [35-37], and more recently in Refs. [38,39], focusing either on a setup of a center-of-mass (CM) energy of several hundred GeV only or on compositeness models. Compared to the other types of colliders, a $\mu p$ collider has its unique advantages. First, while synchrotron radiation prevents a circular electron beam from obtaining high energies, this issue is much more tamed for a muon beam, allowing a $\mu p$ collider to achieve a much higher center-of-mass energy and thus much larger scattering cross sections in general than an electron-proton collider. Further, a $\mu p$ collider shares the upside of an $e p$ collider such that BSM studies on these types of machines usually suffer from smaller QCD backgrounds, than at $p p$ collisions. Moreover, with multi-TeV CM energies, a $\mu p$ collider could produce $\mathrm{TeV}$-scale new particles on shell, which is, however, more difficult to achieve at, e.g., a multi$\mathrm{TeV}$ muon collider.

There are admittedly downsides of a $\mu p$ collider. Notably muons are short-lived. This requires a sufficiently large acceleration for the muon beam so that the muons reach the interaction point before decaying and a careful examination of the beam-induced background (BIB). As Ref. [33] pointed out, the BIB can be reduced by a large extent if the signal final-state particles are largely boosted towards the other beam side. ${ }^{2}$ As we will see, because of the proton parton distribution, this is indeed the case for $\mu p$ collisions even if the proton beam energy is one order of magnitude larger than the muon one.

\footnotetext{
${ }^{2}$ This strategy is, however, inapplicable for muon colliders which suffer from the BIB issue on both sides of the beams.
} 
Given the discussion of $\mu p$ collisions above, one can easily see that multi- $\mathrm{TeV} \mu p$ colliders can probe a much higher scale in deep-inelastic scattering than other collider experiments such as the $\mathrm{LHeC}$. For instance, with a $\mathrm{CM}$ energy of $5 \mathrm{TeV}$, the largest potential reach in momentum squared transfer, $Q^{2}$, can be of order $10^{7} \mathrm{GeV}^{2}$. In the present paper, however, we will focus on studying the potential of $\mu p$ colliders in probing BSM physics.

The organization of this work is as follows. In Sec. II we introduce the relevant parameters of the two tentative $\mu p$ collider setups we propose. We then study in detail in Secs. III-V, the sensitivity reach of these potential experiments in Higgs coupling measurements, R-parity-violating supersymmetry, and finally heavy new physics (NP) in the muon $g-2$. We summarize in Sec. VI.

\section{COLLIDER SETUPS}

In this work we focus on two possible beam combinations: (1) " $\mu p-1$ " with $E_{p}=7 \mathrm{TeV}$ and $E_{\mu^{-}}=1 \mathrm{TeV}$, and (2) " $\mu p-2$ " with $E_{p}=50 \mathrm{TeV}$ and $E_{\mu^{-}}=3 \mathrm{TeV}$. The proton beam energies are in agreement with the HLLHC and FCC, while the muon energies are inspired from the current discussion on $\mathrm{TeV}$-scale muon colliders.

Estimates on the instantaneous luminosity at muonproton colliders were performed in the past $[35,40,41]$. In general, realistic estimates for the luminosity given the current technologies should be at the order of $10^{33} \mathrm{~cm}^{-2} \mathrm{~s}^{-1}$, which we assume for $\mu p-1$. As for $\mu p-2$ which is supposed to be an upgrade of $\mu p-1$, we take a slightly optimistic value of $10^{34} \mathrm{~cm}^{-2} \mathrm{~s}^{-1}$. For the lifespan of these experiments, we take as a benchmark operation time $10^{7} \mathrm{~s} /$ year for 10 years, leading to an integrated luminosity $\mathcal{L}^{\text {int }}$ of $0.1 \mathrm{ab}^{-1}$ and $1 \mathrm{ab}^{-1}$ for $\mu p-1$ and $\mu p-2$, respectively. We summarize these collider parameters in Table I.

\section{HIGGS PRECISION MEASUREMENTS}

One of the utmost tasks in Higgs physics is the precision measurements of the Higgs boson couplings. Here we study the projected uncertainties in the measurement of the Higgs coupling to $b$-quarks at a $\mu p$ collider.

Similar to ep collisions, the Higgs boson at $\mu p$ is produced mainly via the $W W$ and $Z Z$ vector-boson-fusion (VBF) processes. In Table II we list the inclusive production cross sections of the SM Higgs boson at $\mu p-1$, $\mu p-2$, LHeC, FCC-he, and the LHC with $\sqrt{s}=14 \mathrm{TeV}$.

TABLE I. Basic parameters of the two $\mu p$ experiments considered in this work.

\begin{tabular}{lcccc}
\hline \hline Exp. & $E_{p}[\mathrm{TeV}]$ & $E_{\mu^{-}}[\mathrm{TeV}]$ & $\sqrt{s}[\mathrm{TeV}]$ & $\mathcal{L}^{\text {int }\left[\mathrm{ab}^{-1}\right]}$ \\
\hline$\mu p-1$ & 7 & 1 & 5.3 & 0.1 \\
$\mu p-2$ & 50 & 3 & 24.5 & 1 \\
\hline \hline
\end{tabular}

TABLE II. The inclusive cross sections of VBF production of the SM Higgs bosons at various experiments, in pb.

\begin{tabular}{lccccc}
\hline VBF process & $\mu p-1$ & $\mu p-2$ & LHeC & FCC-he & LHC-14 \\
\hline$W W$ & 0.978 & 5.103 & $0.110[13]$ & $0.577[13]$ & \\
ZZ & 0.216 & 1.263 & $0.020[13]$ & $0.127[13]$ & \\
\hline \hline
\end{tabular}

We find that the VBF cross sections at $\mu p-1$ and $\mu p-2$, obtained at leading order with MadGraph5 3.0.2 [42], can be up to about one order of magnitude larger than those at the LHeC and FCC-he, and even comparable to those at the LHC with $\sqrt{s}=14 \mathrm{TeV}$. Here, we choose to focus on the $W W$ VBF process: $p \mu^{-} \rightarrow j \nu_{\mu} h, h \rightarrow b \bar{b}$ because of its larger rate than the $Z Z$ process. The dominant background is the corresponding $W W \mathrm{VBF}$ for $Z$-boson production with $Z \rightarrow b \bar{b}$. We express the measurement uncertainty of the cross section of $p \mu^{-} \rightarrow j \nu_{\mu} b \bar{b}$ as $\Delta \sigma / \sigma=$ $\sqrt{N_{s}+N_{b}} /\left(N_{s}\right)$ including the statistical error only, where $N_{s / b}$ denotes the signal/background event numbers, and perform a cut-based analysis to estimate the sensitivity reach in $\Delta \sigma / \sigma$. We generate the parton-level events with MadGraph5, requiring $p_{T}^{j / b}>5 \mathrm{GeV}$ and $\left|\eta^{j / b}\right|<5.5$. The $p_{T}$ threshold avoids the collinear limit, and the $\left|\eta^{j / b}\right|$ cut corresponds to the geometry of the beam-asymmetric LHeC detector. The parton showering and hadronization for asymmetrical lepton-hadron collisions are properly treated with a patched version of PYTHIA $6.428[43,44]$. Finally we perform jet clustering with FASTJET 3.3.2 [45,46] with the anti- $k_{t}$ algorithm [47], and fast detector simulation with DELPHES 3.4.2 [48]. For the latter we use an LHeCspecific Delphes card which includes the beam asymmetry. For $b$-tagging efficiency we take $75 \%$. The following set of cuts at the reconstructed level are imposed. We first keep only the events with exactly two $b$-jets. In Fig. 1 we show the pseudorapidity distributions of the $b$-jets. We find the produced $b$ 's are peaked at the proton beam side due to the proton parton distributions and expected to allow for BIB reduction. We then select only events where the $b$-jet pair invariant mass, $m_{b b}$, is close to the Higgs mass $125 \mathrm{GeV}$ : $\left|m_{b b}-m_{h}\right|<25 \mathrm{GeV}$, intended to separate the signal and background events. After these event selections, we compute the signal and background event numbers with $N_{s}=\mathcal{L}^{\text {int }} \cdot \sigma\left(p \mu^{-} \rightarrow j \nu_{\mu} h\right) \cdot \operatorname{Br}(h \rightarrow b \bar{b}) \cdot \epsilon_{h b b}^{\mathrm{pr}-\mathrm{cut}} \cdot \epsilon_{\mathrm{cut}}^{\mathrm{sig}}$ and $N_{b}=\mathcal{L}^{\text {int }} \cdot \sigma\left(p \mu^{-} \rightarrow j \nu_{\mu} Z\right) \cdot \operatorname{Br}(Z \rightarrow b \bar{b}) \cdot \epsilon_{Z b b}^{\mathrm{pr}-\mathrm{cut}} \cdot \epsilon_{\mathrm{cut}}^{\mathrm{bgd}}$. $\sigma\left(p \mu^{-} \rightarrow j \nu_{\mu} h\right)$ was given in Table II, $\epsilon_{h b b}^{\mathrm{pr}-\mathrm{cut}}$ and $\epsilon_{Z b b}^{\mathrm{pr}-\mathrm{cut}}$ measure the reduction on the signal and background production cross sections from the parton-level cuts, and $\epsilon_{\text {cut }}^{\text {sig }}$ and $\epsilon_{\text {cut }}^{\text {bgd }}$ are the reconstructed-level cut efficiencies. These are all listed in Table III together with the inclusive cross sections $\sigma\left(p \mu^{-} \rightarrow j \nu_{\mu} Z\right)$. The projected reaches in $\Delta \sigma / \sigma$ at $\mu p-1$ and $\mu p-2$ are thus estimated as $0.97 \%$ and $0.15 \%$, respectively. In order to translate the uncertainties on $\sigma$ to those on the Higgs- $b-\bar{b}$ coupling, $g_{h b b}$, we 


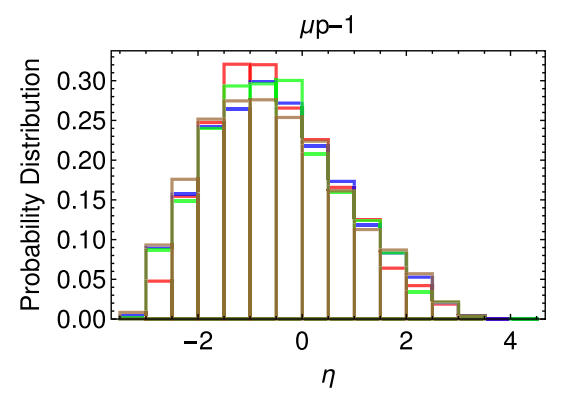

$\square h(b b): b 1$

$\square h(b b): b 2$ $\square \mathrm{Z}(\mathrm{bb}): \mathrm{b} 1$

$\square \mathrm{Z}(\mathrm{bb}): \mathrm{b} 2$

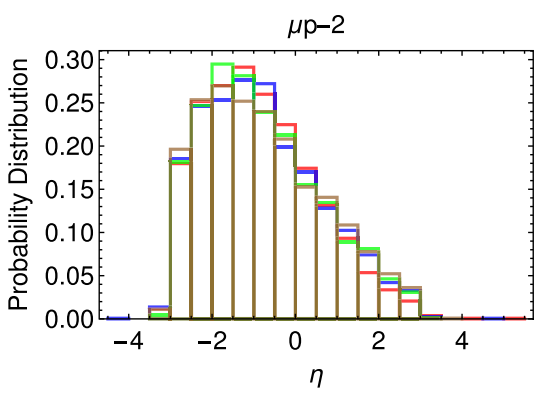

$\square h(b b): b 1$

$\square(b b): b 2$

$\square \mathrm{Z}(\mathrm{bb}): \mathrm{b} 1$

$\square \mathrm{Z}(\mathrm{bb}): \mathrm{b} 2$

FIG. 1. Pseudorapidity distributions of the reconstructed $b$-jets for the signal and background events at $\mu p-1$ and $\mu p-2$.

need to take into account the measurement uncertainties on $\frac{g_{h W W}^{2}}{\Gamma_{h}}$ where $g_{h W W}$ and $\Gamma_{h}$ are the Higgs coupling to the $W$-bosons and the Higgs total decay width. $\Delta\left(\frac{g_{h W W}^{2}}{\Gamma_{h}}\right) / \frac{g_{h W W}^{2}}{\Gamma_{h}}$ can be derived from the uncertainties on the Higgsstrahlung production cross section and its product with $\operatorname{Br}(h \rightarrow W W)$ at the FCC-ee as benchmark values: $\sigma\left(e^{-} e^{+} \rightarrow Z h\right)$ and $\sigma\left(e^{-} e^{+} \rightarrow Z h\right) \cdot \operatorname{Br}(h \rightarrow W W)$. These have been given as $0.4 \%$ and $0.9 \%$ in, e.g., Ref. [49], allowing us to estimate $\Delta\left(\frac{g_{h W W}^{2}}{\Gamma_{h}}\right) / \frac{g_{h W W}^{2}}{\Gamma_{h}} \quad$ as $\quad \sqrt{(0.4 \%)^{2}+(0.9 \%)^{2}}=0.985 \%, \quad$ as $\sigma\left(e^{-} e^{+} \rightarrow Z h\right) \cdot \operatorname{Br}(h \rightarrow W W) / \sigma\left(e^{-} e^{+} \rightarrow Z h\right)=g_{h W W}^{2} / \Gamma_{h}$. Since $\quad \sigma\left(\mu^{-} p \rightarrow \nu_{\mu} j b \bar{b}\right)=\sigma\left(\mu^{-} p \rightarrow \nu_{\mu} j h\right) \cdot \operatorname{Br}(h \rightarrow b \bar{b})=$ $\frac{g_{h W W}^{2} g_{h b b}^{2}}{\Gamma_{h}}$, the uncertainty on $g_{h b b}$ can be computed with $\frac{\Delta g_{h b b}}{g_{h b b}}=\frac{1}{2} \sqrt{\left(\frac{\Delta \sigma}{\sigma}\right)^{2}+\left(\frac{\Delta\left(\frac{g_{h W W}^{2}}{\Gamma_{h}}\right)}{\frac{g_{h W W}^{2}}{\Gamma_{h}}}\right)^{2}}$, which leads to $0.69 \%$ and $0.50 \%$ for $\mu p-1$ and $\mu p-2$, respectively, in comparison with $0.97 \%$ at the $\mathrm{LHeC}$ obtained by a cut-based analysis [50], and $4 \%$ at the CMS experiment with $3 \mathrm{ab}^{-1}$ integrated luminosity $[51,52]$.

TABLE III. Summary of parton-level and reconstructed-level cut efficiencies, and inclusive production cross section of the background process at $\mu p-1$ and $\mu p-2$. The last columns list the final reaches in $\Delta \sigma / \sigma$ and $\Delta g_{h b b} / g_{h b b}$.

\begin{tabular}{lccccccc}
\hline \hline Exp. & $\epsilon_{h b b}^{\mathrm{pr}-\mathrm{cut}}$ & $\sigma\left(p \mu^{-} \rightarrow j \nu_{\mu} Z\right)$ & $\epsilon_{z b b}^{\mathrm{pr}-\mathrm{cut}}$ & $\epsilon_{\mathrm{cut}}^{\mathrm{sig}}$ & $\epsilon_{\mathrm{cut}}^{\mathrm{bgd}}$ & $\frac{\Delta \sigma}{\sigma}$ & $\frac{\Delta g_{h b b}}{g_{h b b}}$ \\
\hline$\mu p-1$ & 0.98 & $4.67 \mathrm{pb}$ & 0.99 & 0.21 & 0.022 & $0.97 \%$ & $0.69 \%$ \\
$\mu p-2$ & 0.91 & $25.1 \mathrm{pb}$ & 0.97 & 0.17 & 0.018 & $0.15 \%$ & $0.50 \%$ \\
\hline \hline
\end{tabular}

We comment that a similar improvement in measuring the other Higgs couplings such as those to the gauge bosons is also expected.

\section{R-PARITY-VIOLATING SUPERSYMMETRY}

Even though no new particles have been discovered at the LHC and TeV-scale lower mass bounds on the squarks and gluinos have been established [54-58], SUSY remains one of the most motivated BSM models. In SUSY, a $Z_{2}$ parity, known as R-parity, is usually assumed, rendering the proton stable and offering the lightest supersymmetric particle as a dark matter candidate. However, it is equally legitimate to consider the R-parity-violating supersymmetry (RPV-SUSY) scenario (see Refs. [59-61] for reviews). The latter, in fact, offers rich phenomenology at colliders. With the broken R-parity, the superpotential of the Minimal Supersymmetric Standard Model (MSSM) is extended with:

$$
\begin{aligned}
W_{\mathrm{RPV}}= & \epsilon_{i} L_{i} \cdot H_{u}+\frac{1}{2} \lambda_{i j k} L_{i} \cdot L_{j} \bar{E}_{k}+\lambda_{i j k}^{\prime} L_{i} \cdot Q_{j} \bar{D}_{k} \\
& +\frac{1}{2} \lambda_{i j k}^{\prime \prime} \bar{U}_{i} \bar{D}_{j} \bar{D}_{k},
\end{aligned}
$$

where the operators in the first line violate lepton numbers and those in the second line violate baryon numbers. Allowing all these terms to be nonvanishing would lead to a too fast proton decay rate unless the couplings are extremely small. For the purpose of this work, we focus on the operator $\lambda_{i j k}^{\prime} L_{i} \cdot Q_{j} \bar{D}_{k}$ while assuming the others are vanishing. ${ }^{3}$ In particular, here the RPV squark is a specific leptoquark which was used to explain a number of flavor anomalies [64-66]. Reference [37] from two decades ago performed an analytic estimate of sensitivity reach at a high-energy muon-proton collider (with $E_{\mu^{ \pm}}=200 \mathrm{GeV}$ and $E_{p}=1 \mathrm{TeV}$ ) to the RPV couplings $\lambda_{2 j 1}^{\prime}$ and $\lambda_{21 k}^{\prime}$ for squark masses below $1 \mathrm{TeV}$. In this work, we focus on one Drell-Yan-like signal process as an example: $p \mu^{-} \rightarrow \mu^{-} u$ (neutral current, or denoted as "NC"), mediated by a rightchiral down-type squark $\tilde{d}_{R k}$ and the RPV coupling $\lambda_{21 k}^{\prime}$, and perform a numerical study with Monte Carlo simulations. As in the previous section we go through the tool chain: MadGraph 5 with a RPV-MSSM UFO model file ${ }^{4}$ and the same parton-level cuts, PYTHIA 6, FASTJET 3, and DELPHES 3 with the LHeC card. Here we switch on only one single RPV coupling $\lambda_{21 k}^{\prime}$, for which the current $\left(36 \mathrm{fb}^{-1}\right)$ and projected $\left(3 \mathrm{ab}^{-1}\right)$ LHC bounds were recast in Ref. [67] from an ATLAS mono-lepton search [68]: $\lambda_{21 k}^{\prime}<0.090 \frac{m_{\tilde{d}_{R k}}}{1 \mathrm{TeV}}+0.014$ and $\lambda_{21 k}^{\prime}<0.053 \frac{m_{\tilde{d}_{R k}}}{1 \mathrm{TeV}}+0.029$.

\footnotetext{
${ }^{3}$ This can be justified by e.g., imposing a baryon triality $B_{3}$ symmetry $[62,63]$.

${ }^{4}$ The model file can be found at https://github.com/ilmonteux/ RPVMSSM_UFO.
} 
The background process is $p \mu^{-} \rightarrow j \mu^{-}$plus zero or one extra jet, for which we perform jet matching and merging. Note that we ignore the subdominant effect from the interference terms. For the cuts on the reconstructed events, we first select events with at least 1 jet. We then specifically require that exactly one muon should be reconstructed. We finally keep only events with the $p_{T}$ sum of the two leading jets, $p_{T}^{j 1}+p_{T}^{j 2}$, larger than certain values (for the events with exactly one jet we take $p_{T}^{j 2}=0$ ). We define the signal significance $S$ as $S=N_{s} / \sqrt{N_{b}}$ and determine the 95\% C.L. (confidence level) exclusion limits at $S=2$, where $N_{s / b}$ labels the signal/background event numbers. The resulting limits on $\lambda_{21 k}^{\prime}$ as a function of $m_{\tilde{d}_{R k}}$ for various $p_{T}$ sum thresholds are presented in Fig. 2, which we overlap in red with the current LHC (solid) and future HLLHC (dashed) bounds [67]. We find that increasing the lower threshold for the $p_{T}$ sum of the two leading jets allows to probe heavier $\tilde{d}_{R k}$. We conclude that $\mu p-1(2)$ may exclude values of $\lambda_{21 k}^{\prime}$ down to $0.02(0.01)$ for $m_{\tilde{d}_{R k}} \sim \mathcal{O}(\mathrm{TeV})$. Compared to the HL-LHC, these $\mu p$ limits in $\lambda_{21 k}^{\prime}$ are stronger by up to more than one order of magnitude for $\tilde{d}_{R k}$ light enough to be produced on shell.
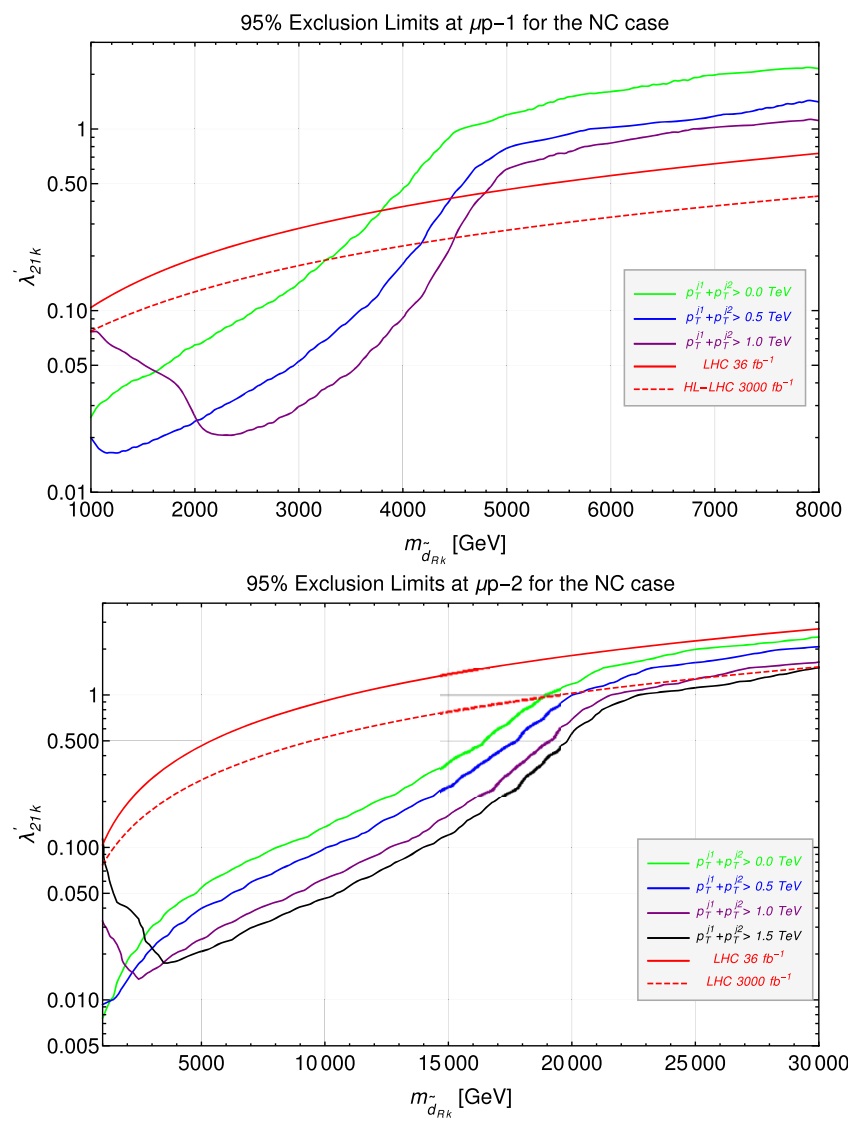

FIG. 2. $95 \%$ C.L. exclusion limits for $\lambda_{21 k}^{\prime}$ vs $m_{\tilde{d}_{R k}}$.
The future hadron-hadron colliders such as the FCC-hh are expected to exclude SUSY squarks up to about $10 \mathrm{TeV}$ [69]. This is comparable to the $\mu p-1 / 2$ considered here. As for future lepton colliders, $e^{-} e^{+}$colliders are expected to perform much worse because of the relatively small center-of-mass energies, while multi-TeV muon colliders have recently been shown to possess huge potential for probing a similar theoretical scenario, i.e., the leptoquarks, possibly excluding the leptoquark mass at the order of $10 \mathrm{TeV}$ [32]. We note that another possible signature with the same mediator and RPV coupling is the charged-current process $p \mu^{-} \rightarrow d \nu_{\mu}$. However, we find that the exclusion limits in this scenario are similar to the $\mathrm{NC}$ results shown in Fig. 2, and hence do not present the results here.

\section{MUON $g-2$ ANOMALY}

One of the main drives for BSM physics has been the muon anomalous magnetic moment since about a decade ago. With the latest world consensus on the SM computation of $a_{\mu} \equiv\left(g_{\mu}-2\right) / 2$ [70] combined with the experimental results published by the E821 collaboration at BNL [71] and recently by the Fermilab-based Muon $g-2$ experiment [72], we are now faced with a discrepancy of $\sim 4.2 \sigma$ in $a_{\mu}: \Delta a_{\mu}=a_{\mu}^{\mathrm{exp}}-a_{\mu}^{\mathrm{SM}}=251(59) \times 10^{-11.5}$. One natural explanation could be weakly interacting NP appearing at the EW scale. However, given the nonobservation of NP at the LHC so far, two other possibilities might be more relevant: (1) light NP below the GeV scale interacting feebly with the SM, and (2) much heavier NP (above the $\mathrm{TeV}$ scale) strongly coupled to the SM particles. In this work, we consider the latter possibility. If the NP scale, $\Lambda$, is much higher than the EW scale, i.e., $\Lambda \gg 1 \mathrm{TeV}$, we can describe physics at energies much below $\Lambda$ with the framework of the Standard Model Effective Field Theory (SMEFT) [74-77], of which the operators up to dim-6 relevant to $a_{\mu}$ are

$$
\begin{aligned}
\mathcal{L} \ni & \frac{C_{e B}^{\mu}}{\Lambda^{2}}\left(\bar{\mu}_{L} \sigma^{\mu \nu} \mu_{R}\right) H B_{\mu \nu}+\frac{C_{e W}^{l}}{\Lambda^{2}}\left(\bar{\mu}_{L} \sigma^{\mu \nu} \mu_{R}\right) \sigma^{i} H W_{\mu \nu}^{i} \\
& +\frac{C_{T}^{\mu}}{\Lambda^{2}}\left(\bar{\mu}_{L}^{a} \sigma_{\mu \nu} \mu_{R}\right) \epsilon_{a b}\left(\bar{Q}_{L}^{b} \sigma^{\mu \nu} u_{R}\right)+\text { H.c. }
\end{aligned}
$$

The NP contributions to $a_{\mu}$ stem directly from the operator $\left(\bar{\mu}_{L} \sigma_{\mu \nu} \mu_{R}\right) H F^{\mu \nu}$, which may be induced by the operators in Eq. (2) at tree or one-loop level. Their corrections to $a_{\mu}$ can be expressed as follows:

\footnotetext{
${ }^{5}$ There is a controversy arising from a new lattice calculation [73] which shows a larger hadronic vacuum polarization contribution, such that the total SM contribution to the muon $g-2$ is within $1 \sigma$ of the experimental value.
} 


$$
\begin{aligned}
\Delta a_{\mu} \simeq & \frac{4 m_{\mu} v}{e \sqrt{2} \Lambda^{2}}\left(C_{e \gamma}^{\mu}-\frac{3 \alpha}{2 \pi} \frac{c_{W}^{2}-s_{W}^{2}}{c_{W} s_{W}} C_{e Z}^{\mu} \log \frac{\Lambda}{m_{Z}}\right) \\
& -\sum_{q=c, t} \frac{4 m_{\mu} m_{q}}{\pi^{2}} \frac{C_{T}^{\mu q}}{\Lambda^{2}} \log \frac{\Lambda}{m_{q}},
\end{aligned}
$$

where $v=246 \mathrm{GeV}$ is the SM Higgs vacuum expectation value, $s_{W}$ and $c_{W}$ are sine and cosine of the Weinberg angle, and $C_{e \gamma}=c_{W} C_{e B}-s_{W} C_{e W}$ and $C_{e Z}=-s_{W} C_{e B}-c_{W} C_{e W}$.

At a $\mu p$ collider, only a limited set of these operators can be tested. To start with, $C_{e \gamma}^{\mu}$ can be probed, either by considering the photon content inside the protons scattering an incoming muon, or by studying rare Higgs decays into a pair of muons and a photon. We find the former possibility, suppressed by the parton distribution function of the photon in the protons, is insensitive to new physics that is sufficiently small to explaining the muon $g-2$ anomaly. As for the rare Higgs decay, the decay branching ratio of the Higgs into $\mu^{+} \mu^{-} \gamma$ should be at the order of $\sim 10^{-13}$ in order to test $\Delta a_{\mu} \sim 3 \times 10^{-9}$ [24]. However, at both $\mu p-1$ and $\mu p-2$ the production rates of the SM Higgs bosons are estimated to be roughly $10^{5}$ and $5 \times 10^{6}$ (see Table II), with $0.1 \mathrm{ab}^{-1}$ and $1 \mathrm{ab}^{-1}$ integrated luminosities, respectively, which are far from sufficient for probing a branching ratio of $10^{-13}$. Consequently the only operator that could be confronted for $\Delta a_{\mu} \sim 3 \times 10^{-9}$ at $\mu p-1$ and $\mu p-2$ is $C_{T}^{\mu c}$, with the parton-level process $\mu^{-} c \rightarrow \mu^{-} c$ and its $\bar{c}$ counterpart. The corresponding background is $\mu^{-} p \rightarrow \mu^{-} j$. Note that the unitarity constraint requires that $\Lambda \lesssim 10 \mathrm{TeV}$ for this operator.

To explore the heavy NP in the muon $g-2$ at $\mu p-1$ and $\mu p-2$, we perform truth-level Monte Carlo simulations with the event generator MadGraph5 and the model package SMEFTsim [78,79], with the parton-level cuts $p_{T}^{j}>$ $5 \mathrm{GeV}$ and $\left|\eta^{j}\right|<5.5$. The computed cross sections for the signal and background processes are given in Table IV, assuming the contributions arise solely from the single SMEFT operator $C_{T}^{\mu c}$. Therefore we can easily obtain the signal and background event numbers, and hence the $2 \sigma$ exclusion limits on $C_{T}^{\mu c} / \Lambda^{2}$. To convert these limits into those on $\left|\Delta a_{\mu}\right|$, we take $\Lambda=10 \mathrm{TeV}$ for the logarithmic function in the last term of Eq. (3), reaching $\left|\Delta a_{\mu}\right|=\frac{4 m_{\mu} m_{c}}{\pi^{2}}\left|\frac{C_{T}^{\mu c}}{\Lambda^{2}}\right| \log \frac{10 \mathrm{TeV}}{m_{c}}$. The exclusion limits on $\left|\Delta a_{\mu}\right|$ are given in the last column of Table IV: $1.13 \times 10^{-8}$ and $9.10 \times 10^{-10}$. We conclude that in the limit of vanishing contributions from the other operators, the low-energy
TABLE IV. Cross sections for the background ( $\left.\sigma^{\text {bgd }}\right)$ and signal $\left(\sigma^{\text {sig }}\right)$ events, as well as the integrated luminosities and the expected exclusion limits of $\left|\Delta a_{\mu}\right|$, at $\mu p-1$ and $\mu p-2$.

\begin{tabular}{lccc}
\hline \hline & $\sigma^{\mathrm{bgd}}[\mathrm{pb}]$ & $\sigma^{\mathrm{sig}}\left(\frac{\Lambda^{2}}{100 \mathrm{TeV}^{2} C_{T}^{\mu}}\right)^{2}[\mathrm{pb}]$ & $\left|\Delta a_{\mu}\right|$ \\
\hline$\mu p-1$ & 120 & $1.29 \times 10^{-2}$ & $1.13 \times 10^{-8}$ \\
$\mu p-2$ & 30 & $3.15 \times 10^{-1}$ & $9.10 \times 10^{-10}$ \\
\hline \hline
\end{tabular}

effects from the high-scale NP associated with the tensor operator $C_{T}^{\mu c}$ that would be small enough to explain the muon $g-2$ anomaly can be probed at $\mu p-2$. In order to make $\mu p-1$ sensitive enough, further improvements on, e.g., luminosity and search strategies, should be implemented.

We note that since only $\Lambda \lesssim 10 \mathrm{TeV}$ is valid for the considered operator, it is necessary to check whether the typical hard-interaction $\mathrm{CM}$ energies for the signal are lower than $10 \mathrm{TeV}$. We find that for $\mu p-1, \sim 100 \%$ of the events have the invariant mass $m_{\mu c}$ of the outgoing muon and $c$ quark $m_{\mu c}<5 \mathrm{TeV}$, and for $\mu p-2$ it is about $\sim 70 \%$ despite the much higher $\mathrm{CM}$ energy.

\section{CONCLUSIONS}

In this work we have proposed a muon-proton collider with two tentative configurations. We performed numerical simulations to investigate the physics potential of $\mu p-1$ and $\mu p-2$ in both Higgs precision measurement and search for BSM physics. Taking as benchmark physics cases the Higgs coupling to $b$-quarks, R-parity-violating MSSM, and heavy new physics in the muon $g-2$, we conclude that a multi-TeV muon-proton collider with $0.1-1 \mathrm{ab}^{-1}$ integrated luminosities could show better performance than both current and future collider experiments. Besides the physics scenarios studied here, we expect that this type of machine can also excel in other aspects of the SM precision measurements and BSM physics searches. We believe this work could motivate more studies of TeVscale muon-proton colliders in the high-energy physics community.

\section{ACKNOWLEDGMENTS}

We would like to thank Florian Domingo and Jong Soo Kim for useful discussions. This work was supported by MoST with Grants No. MoST-109-2811-M-007-509 and No. 107-2112-M-007-029-MY3. 
[1] G. Aad et al. (ATLAS Collaboration), Observation of a new particle in the search for the Standard Model Higgs boson with the ATLAS detector at the LHC, Phys. Lett. B 716, 1 (2012).

[2] S. Chatrchyan et al. (CMS Collaboration), Observation of a new boson at a mass of $125 \mathrm{GeV}$ with the CMS experiment at the LHC, Phys. Lett. B 716, 30 (2012).

[3] H. P. Nilles, Supersymmetry, supergravity and particle physics, Phys. Rep. 110, 1 (1984).

[4] S. P. Martin, A Supersymmetry primer, Adv. Ser. Dir. High Energy Phys. 21, 1 (2010).

[5] G. Aarons et al. (ILC Collaboration), International Linear collider reference design report volume 2: Physics at the ILC, arXiv:0709.1893.

[6] T. Behnke et al., The international linear collider technical design report-volume 1: Executive summary, arXiv: 1306.6327.

[7] T. Charles et al. (CLICdp, CLIC Collaborations), The compact linear collider (CLIC)—2018 summary report, arXiv:1812.06018.

[8] A. Abada et al. (FCC Collaboration), FCC-ee: The Lepton Collider: Future circular collider conceptual design report volume 2, Eur. Phys. J. Special Topics 228, 261 (2019).

[9] M. Dong et al. (CEPC Study Group Collaboration), CEPC conceptual design report: Volume 2-Physics, arXiv: 1811.10545.

[10] M. Klein, The large hadron electron collider project, in 17th International Workshop on Deep-Inelastic Scattering and Related Subjects (2009), p. 236, https://inspirehep.net/ literature/829172.

[11] J. Abelleira Fernandez et al. (LHeC Study Group Collaboration), A large hadron electron collider at CERN: Report on the physics and design concepts for machine and detector, J. Phys. G 39, 075001 (2012).

[12] O. Bruening and M. Klein, The large hadron electron collider, Mod. Phys. Lett. A 28, 1330011 (2013).

[13] P. Agostini et al. (LHeC, FCC-he Study Group Collaborations), The large hadron-electron collider at the HL-LHC, arXiv:2007.14491.

[14] A. Abada et al. (FCC Collaboration), FCC physics opportunities: Future circular collider conceptual design report volume 1, Eur. Phys. J. C 79, 474 (2019).

[15] A. Abada et al. (FCC Collaboration), FCC-hh: The hadron collider: Future circular collider conceptual design report volume 3, Eur. Phys. J. Special Topics 228, 755 (2019).

[16] X. Cid Vidal et al., Report from working group 3: Beyond the standard model physics at the HL-LHC and HE-LHC, CERN Yellow Rep. Monogr. 7, 585 (2019).

[17] A. Abada et al. (FCC Collaboration), HE-LHC: The highenergy large hadron collider: Future circular collider conceptual design report volume 4, Eur. Phys. J. Special Topics 228, 1109 (2019).

[18] P. Azzi et al., Report from working group 1: Standard model physics at the HL-LHC and HE-LHC, CERN Yellow Rep. Monogr. 7, 1 (2019).

[19] J. Tang et al., Concept for a future super proton-proton collider, arXiv:1507.03224.

[20] T. Han and Z. Liu, Potential precision of a direct measurement of the Higgs boson total width at a muon collider, Phys. Rev. D 87, 033007 (2013).
[21] J. P. Delahaye et al., Muon colliders, arXiv:1901.06150.

[22] A. Costantini, F. De Lillo, F. Maltoni, L. Mantani, O. Mattelaer, R. Ruiz, and X. Zhao, Vector boson fusion at multi-TeV muon colliders, J. High Energy Phys. 09 (2020) 080.

[23] T. Han, Z. Liu, L.-T. Wang, and X. Wang, WIMPs at high energy muon colliders, Phys. Rev. D 103, 075004 (2021).

[24] D. Buttazzo and P. Paradisi, Probing the muon g-2 anomaly at a Muon Collider, arXiv:2012.02769.

[25] W. Yin and M. Yamaguchi, Muon $g-2$ at multi-TeV muon collider, arXiv:2012.03928.

[26] R. Capdevilla, D. Curtin, Y. Kahn, and G. Krnjaic, A guaranteed discovery at future muon colliders, Phys. Rev. D 103, 075028 (2021).

[27] T. Han, D. Liu, I. Low, and X. Wang, Electroweak couplings of the Higgs boson at a multi-TeV muon collider, Phys. Rev. D 103, 013002 (2021).

[28] K. Long, D. Lucchesi, M. A. Palmer, N. Pastrone, D. Schulte, and V. Shiltsev, Muon colliders: Opening new horizons for particle physics, Nat. Phys. 17, 289 (2021).

[29] G.-y. Huang, F. S. Queiroz, and W. Rodejohann, Gauged $L_{\mu}-L_{\tau}$ at a muon collider, arXiv:2101.04956.

[30] R. Capdevilla, D. Curtin, Y. Kahn, and G. Krnjaic, A nolose theorem for discovering the new physics of $(g-2)_{\mu}$ at muon colliders, arXiv:2101.10334.

[31] W. Liu and K.-P. Xie, Probing electroweak phase transition with multi-TeV muon colliders and gravitational waves, J. High Energy Phys. 04 (2021) 015.

[32] P. Asadi, R. Capdevilla, C. Cesarotti, and S. Homiller, Searching for leptoquarks at future muon colliders, arXiv: 2104.05720.

[33] M. Lu, A. M. Levin, C. Li, A. Agapitos, Q. Li, F. Meng, S. Qian, J. Xiao, T. Yang, and M. Frank, The physics case for an electron-muon collider, Adv. High Energy Phys. 2021, 6693618 (2021).

[34] F. Bossi and P. Ciafaloni, Lepton flavor violation at muon-electron colliders, J. High Energy Phys. 10 (2020) 033.

[35] V. D. Shiltsev, An Asymmetric muon-proton collider: Luminosity consideration, Conf. Proc. C 970512, 420 (1997), https://www.osti.gov/biblio/16114.

[36] K.-m. Cheung, Muon proton colliders: Leptoquarks, contact interactions and extra dimensions, AIP Conf. Proc. 542, 160 (2000).

[37] M. Carena, D. Choudhury, C. Quigg, and S. Raychaudhuri, Study of R-parity violation at a muon proton collider, Phys. Rev. D 62, 095010 (2000).

[38] A. Caliskan, S. Kara, and A. Ozansoy, Excited muon searches at the FCC based muon-hadron colliders, Adv. High Energy Phys. 2017, 1540243 (2017).

[39] Y. C. Acar, U. Kaya, and B. B. Oner, Resonant production of color octet muons at Future Circular Collider-based muon-proton colliders, Chin. Phys. C 42, 083108 (2018).

[40] I. Ginzburg, Physics at future e p, gamma p (linac-ring) and mu p colliders, Turk. J. Phys. 22, 607 (1998), https:// inspirehep.net/literature/479511.

[41] U. Kaya, B. Ketenoglu, S. Sultansoy, and F. Zimmermann, Main parameters of HL-LHC and HE-LHC based mu-p colliders, arXiv:1905.05564.

[42] J. Alwall, R. Frederix, S. Frixione, V. Hirschi, F. Maltoni, O. Mattelaer, H.-S. Shao, T. Stelzer, P. Torrielli, and M. Zaro, 
The automated computation of tree-level and next-toleading order differential cross sections, and their matching to parton shower simulations, J. High Energy Phys. 07 (2014) 079.

[43] T. Sjostrand, S. Mrenna, and P.Z. Skands, PYTHIA 6.4 physics and manual, J. High Energy Phys. 05 (2006) 026.

[44] U. Klein (private communication).

[45] M. Cacciari, G. P. Salam, and G. Soyez, FASTJET user manual, Eur. Phys. J. C 72, 1896 (2012).

[46] M. Cacciari and G. P. Salam, Dispelling the $N^{3}$ myth for the $k_{t}$ jet-finder, Phys. Lett. B 641, 57 (2006).

[47] M. Cacciari, G. P. Salam, and G. Soyez, The anti- $k_{t}$ jet clustering algorithm, J. High Energy Phys. 04 (2008) 063.

[48] J. de Favereau, C. Delaere, P. Demin, A. Giammanco, V. Lemaître, A. Mertens, and M. Selvaggi (DELPHES 3 Collaboration), DELPHES 3, A modular framework for fast simulation of a generic collider experiment, J. High Energy Phys. 02 (2014) 057.

[49] M. Ruan, Higgs measurement at $e^{+} e^{-}$circular colliders, Nucl. Part. Phys. Proc. 273-275, 857 (2016).

[50] M. Tanaka, SM Higgs at the LHeC, https://indico.cern.ch/ event/568360/contributions/2523555/attachments/1440097/ 2216668/mtanaka_DIS2017_SMhiggs_final.pdf.

[51] CMS, Projected performance of an upgraded CMS detector at the LHC and HL-LHC: Contribution to the snowmass process, arXiv:1307.7135.

[52] M. Cepeda et al., Report from working group 2: Higgs physics at the HL-LHC and HE-LHC, CERN Yellow Rep. Monogr. 7, 221 (2019).

[53] CERN Yellow Report Page At 1314 TeV 2014, https://twiki .cern.ch/twiki/bin/view/LHCPhysics/CERNYellowReport PageAt1314TeV2014.

[54] M. Aaboud et al. (ATLAS Collaboration), Search for photonic signatures of gauge-mediated supersymmetry in $13 \mathrm{TeV} p p$ collisions with the ATLAS detector, Phys. Rev. D 97, 092006 (2018).

[55] A. M. Sirunyan et al. (CMS Collaboration), Search for gauge-mediated supersymmetry in events with at least one photon and missing transverse momentum in pp collisions at $\sqrt{s}=13 \mathrm{TeV}$, Phys. Lett. B 780, 118 (2018).

[56] A. M. Sirunyan et al. (CMS Collaboration), Search for supersymmetry in final states with photons and missing transverse momentum in proton-proton collisions at $13 \mathrm{TeV}$, J. High Energy Phys. 06 (2019) 143.

[57] A. M. Sirunyan et al. (CMS Collaboration), Search for supersymmetry in proton-proton collisions at $13 \mathrm{TeV}$ in final states with jets and missing transverse momentum, J. High Energy Phys. 10 (2019) 244.

[58] G. Aad et al. (ATLAS Collaboration), Search for new phenomena in final states with large jet multiplicities and missing transverse momentum using $\sqrt{s}=13 \mathrm{TeV}$ protonproton collisions recorded by ATLAS in Run 2 of the LHC, J. High Energy Phys. 10 (2020) 062.

[59] H. K. Dreiner, An Introduction to explicit R-parity violation, Adv. Ser. Dir. High Energy Phys. 21, 565 (2010).

[60] R. Barbier et al., R-parity violating supersymmetry, Phys. Rep. 420, 1 (2005).

[61] R. N. Mohapatra, Supersymmetry and R-parity: An overview, Phys. Scr. 90, 088004 (2015).
[62] L. E. Ibanez and G. G. Ross, Discrete gauge symmetries and the origin of baryon and lepton number conservation in supersymmetric versions of the standard model, Nucl. Phys. B368, 3 (1992).

[63] H. K. Dreiner, M. Hanussek, and C. Luhn, What is the discrete gauge symmetry of the R-parity violating MSSM?, Phys. Rev. D 86, 055012 (2012).

[64] E. Coluccio Leskow, G. D’Ambrosio, A. Crivellin, and D. Müller, $(g-2) \mu$, lepton flavor violation, and $Z$ decays with leptoquarks: Correlations and future prospects, Phys. Rev. D 95, 055018 (2017).

[65] A. Crivellin, C. Greub, D. Müller, and F. Saturnino, Scalar leptoquarks in leptonic processes, J. High Energy Phys. 02 (2021) 182.

[66] A. Crivellin, D. Mueller, and F. Saturnino, Correlating $h \rightarrow$ $\mu^{+} \mu^{-}$to the anomalous magnetic moment of the muon via leptoquarks, arXiv:2008.02643.

[67] S. Bansal, A. Delgado, C. Kolda, and M. Quiros, Limits on R-parity-violating couplings from Drell-Yan processes at the LHC, Phys. Rev. D 99, 093008 (2019).

[68] M. Aaboud et al. (ATLAS Collaboration), Search for a new heavy gauge boson resonance decaying into a lepton and missing transverse momentum in $36 \mathrm{fb}^{-1}$ of $p p$ collisions at $\sqrt{s}=13 \mathrm{TeV}$ with the ATLAS experiment, Eur. Phys. J. C 78, 401 (2018).

[69] J. I. Loukas Gouskos and A. Sung, Top squark searches at $100 \mathrm{TeV}$, https://indico.cern.ch/event/704428/contributions/ 2957539/attachments/1627650/2592608/fcc-hh-susystop20180315-lg.pdf.

[70] T. Aoyama et al., The anomalous magnetic moment of the muon in the Standard Model, Phys. Rep. 887, 1 (2020).

[71] G. Bennett et al. (Muon g-2 Collaboration), Final report of the muon E821 anomalous magnetic moment measurement at BNL, Phys. Rev. D 73, 072003 (2006).

[72] B. Abi et al. (Muon g-2 Collaboration), Measurement of the Positive Muon Anomalous Magnetic Moment to $0.46 \mathrm{ppm}$, Phys. Rev. Lett. 126, 141801 (2021).

[73] S. Borsanyi et al., Leading hadronic contribution to the muon 2 magnetic moment from lattice QCD, Nature (London) 593, 51 (2021).

[74] W. Buchmuller and D. Wyler, Effective lagrangian analysis of new interactions and flavor conservation, Nucl. Phys. B268, 621 (1986).

[75] B. Grzadkowski, M. Iskrzynski, M. Misiak, and J. Rosiek, Dimension-six terms in the standard model lagrangian, J. High Energy Phys. 10 (2010) 085.

[76] E. E. Jenkins, A. V. Manohar, and M. Trott, Renormalization group evolution of the standard model dimension six operators II: Yukawa dependence, J. High Energy Phys. 01 (2014) 035.

[77] R. Alonso, E. E. Jenkins, A. V. Manohar, and M. Trott, Renormalization group evolution of the standard model dimension six operators III: Gauge coupling dependence and phenomenology, J. High Energy Phys. 04 (2014) 159.

[78] I. Brivio, Y. Jiang, and M. Trott, The SMEFTsim package, theory and tools, J. High Energy Phys. 12 (2017) 070.

[79] I. Brivio, SMEFTsim 3.0 — a practical guide, J. High Energy Phys. 04 (2021) 073. 